\title{
Resumption of anticoagulation therapy after spontaneous intracerebral hemorrhage with patients mechanical heart valves
}

\author{
Rubin Luo ${ }^{1}$, Zhao Zhai ${ }^{2}$, Qin $\mathrm{Wu}^{2}$, Kan Chen ${ }^{2}$, Huixing $\mathbf{Y i}^{3}$ \\ ${ }^{1}$ Department of Surgical Intensive Care Unit, The Second Affiliated Hospital of Zhejiang University School of Medicine, Hangzhou, China; \\ ${ }^{2}$ Department of Emergency Medicine, The First Affiliated Hospital of Gannan Medical University, Ganzhou, China; ${ }^{3}$ Department of Intensive Care \\ Unit, The Fourth Affiliated Hospital of Jiangsu University, Zhenjiang, China \\ Contributions: (I) Conception and design: R Luo; (II) Administrative support: H Yi; (III) Provision of study materials or patients: R Luo; (IV) \\ Collection and assembly of data: R Luo; (V) Data analysis and interpretation: R Luo; (VI) Manuscript writing: All authors; (VII) Final approval of \\ manuscript: All authors. \\ Correspondence to: Huixing Yi, MD. Department of Intensive Care Unit, The Fourth Affiliated Hospital of Jiangsu University, 20 Zhengdong Road, \\ Zhenjiang 212001, China. Email: yhxhqypy@163.com.
}

Background: Patients with mechanical heart valves are usually maintained on anticoagulation therapy. However, after a spontaneous intracerebral hemorrhage event, administration of anticoagulants is temporarily ceased, and it remains unclear when to restart anticoagulation therapy.

Methods: A cohort study was conducted to investigate the optimal time for restarting anticoagulation in patients with mechanical heart valves after spontaneous intracerebral hemorrhage. All patients with mechanical valves who experienced spontaneous cerebral hemorrhage and were admitted to the Second Affiliated Hospital of the Zhejiang University Medical School between 2013 and 2018 were retrospectively enrolled in this study. The patient electronic medical records were reviewed and the correlation between the time of restarting anticoagulation (within 3 days or more than 3 days after hemorrhage) and patient prognosis was assessed.

Results: A total of 40 patients with mechanical heart valves who experienced spontaneous cerebral hemorrhage were enrolled in this study. All patients were given oral warfarin anticoagulant therapy prior to admission (1.5-3.25 mg). After admission, patients were administered fresh frozen plasma and/or vitamin $\mathrm{K} 1$ to reverse anticoagulation. Out of the 16 patients (40\%) who underwent surgical intervention, 4 died from cerebral hemorrhage deterioration during the hospital stay and did not restart anticoagulant therapy. Anticoagulant therapy was resumed within 3 days for 18 patients and more than three days after hemorrhage for the other 18 patients. After discharge, patients were followed up for 12 months or more. Unfortunately, during this period, $17 \%$ of patients $(6 / 36)$ died.

Conclusions: Definitive hemostatic measures can be as an important factor in the clinical resumption of anticoagulation. Halting anticoagulant therapy for 3 to 7 days may be safe. It is recommended that low molecular heparin be administered within 3 days as a bridge treatment, combine with warfarin anticoagulant therapy within 1 week after hemorrhage.

Keywords: Anticoagulation; bleeding; mechanical heart valve; cerebral hemorrhage; thromboembolism

Submitted Oct 21, 2021. Accepted for publication Jan 12, 2022.

doi: 10.21037/atm-21-6848

View this article at: https://dx.doi.org/10.21037/atm-21-6848 


\section{Introduction}

Anticoagulation therapy is recommended in all patients with mechanical valvular replacement due to the significant associated risks of thromboembolism and valve dysfunction (1). Anticoagulation management is dependent on thrombogenicity, the site of valve replacement, and the associated risk factors of the valve (2). Bleeding events related to long-term oral anticoagulant therapy, for coagulation was not monitored during warfarin administration, the incidence of intracerebral hemorrhage (nearly $0.3-0.7 \%$ per year), are the most common complications in patients with mechanical valves (3). However, the temporary cessation of anticoagulant therapy may lead to the risk of valve thrombosis and stuck valve. Therefore, balancing the risk of recurrent cerebral hemorrhage and thromboembolism is crucial for such patients $(4,5)$. Unfortunately, currently there is no consensus or guideline on when to restart anticoagulation therapy after cerebral hemorrhage in patients with mechanical valves.

This retrospective study analyzed the diagnosis, treatment, and prognosis of restarting anticoagulation therapy in patients with mechanical heart valves after spontaneous cerebral hemorrhage. This is the first cohort study of resumption of anticoagulation therapy reported from China.

We present the following article in accordance with the STROBE reporting checklist (available at https://atm. amegroups.com/article/view/10.21037/atm-21-6848/rc).

\section{Methods}

The electronic medical records of 40 patients with mechanical valves who experienced spontaneous cerebral hemorrhage and were admitted to the Second Affiliated Hospital of Zhejiang University School of Medicine between 2013 to 2018 were retrospectively analyzed. All procedures performed in this study involving human participants were in accordance with the Declaration of Helsinki (as revised in 2013). The study was approved by ethics committee of the Second Affiliated Hospital of Zhejiang University School of Medicine (No. 2020318). Individual consent for this retrospective analysis was waived. A total of 4 patients died during hospitalization and anticoagulation therapy was resumed in 36 patients to prevent mechanical valve thromboembolism. A head computed tomography (CT) examination and surgery was performed on the first day after bleeding to confirm that the bleeding was stable or that the hematoma had been removed. Resumption of anticoagulation therapy was at the discretion of the neurosurgical doctor and was doseadjusted based on the international normalized ratio (INR) standard.

Patients were divided into 2 groups based on whether anticoagulation therapy was resumed within 3 days after cerebral hemorrhage or more than 3 days after hemorrhage. All patients were followed-up for 12 months or more.

Patient data, including age, gender, type of mechanical heart valve, replacement time, replacement site, underlying disease, anticoagulant drugs at admission, type of cerebral hemorrhage, treatment mode, time without anticoagulant, length of hospital stay, and mortality, were collated and reviewed.

\section{Statistical analysis}

The SPSS25 software was used for statistical analysis and the analysis of variance (ANOVA) single factor test was used to compare 2 groups. A P value $<0.05$ was considered statistically significant.

\section{Results}

\section{General information}

During the 5-year study timeframe, there were 40 patients with mechanical heart valve replacement who experienced spontaneous cerebral hemorrhage. All patients were receiving oral warfarin anticoagulant therapy prior to admission $(1.5-3.25 \mathrm{mg})$. Upon admission, patients were immediately administered fresh frozen plasma and vitamin $\mathrm{K} 1$ to eliminate the effect of anticoagulation. A total of 16 patients underwent surgical intervention and 24 patients were managed with conservative treatment. Unfortunately, there were 4 in-hospital deaths due to deterioration of the cerebral hemorrhage and thus, anticoagulant therapy could not be restarted in these patients.

The clinical characteristics of the remaining 36 patients are shown in Table 1. There was no significant difference between male and female patients. The age of onset ranged from 50-70 years old. Cerebral hemorrhage occurred within 10 years of valve replacement in 30 patients and after 20 years in a 29 -year-old young man. A total of 16 patients presented with more than 2 chronic diseases before admission, predominantly hypertension, atrial fibrillation, and diabetes. No basic chronic disease was noted in 9 patients. 
Table 1 Baseline patient characteristic

\begin{tabular}{|c|c|c|c|c|}
\hline Variables & Overall $(n=36)$ & $\begin{array}{l}\text { Restart anticoagulation } \\
\text { within } 3 \text { days }(n=18)\end{array}$ & $\begin{array}{l}\text { Restart anticoagulation after } \\
\text { more than } 3 \text { days }(n=18)\end{array}$ & $P$ value \\
\hline Age (years), median [IQR] & $60[51-67]$ & $61[51-68]$ & $61[52-63]$ & 0.614 \\
\hline Female, $\mathrm{n}[\%]$ & $18[50]$ & $10[56]$ & $8[44]$ & 0.519 \\
\hline $\begin{array}{l}\text { Duration from valve replacement to } \mathrm{ICH} \\
\text { onset (years), median [IQR] }\end{array}$ & 5 [2-9] & $7[3-10]$ & 3 [2-9] & 0.338 \\
\hline Aortic & $10[28]$ & $5[28]$ & $5[28]$ & \\
\hline Mitral & $17[47]$ & 9 [50] & $8[44]$ & \\
\hline Both & 9 [25] & 4 [22] & 5 [28] & \\
\hline Diabetes mellitus & $7[19]$ & $3[17]$ & $4[22]$ & 0.684 \\
\hline Other diseases* & 14 [39] & $9[50]$ & $5[28]$ & 0.181 \\
\hline Pre-admission antithrombotic, $\mathrm{n}$ [\%] & & & & 1.000 \\
\hline Warfarin & 32 [89] & 16 [89] & 16 [89] & \\
\hline Warfarin plus antiplatelet agent & $4[11]$ & $2[11]$ & $2[11]$ & \\
\hline Location, n [\%] & & & & 0.261 \\
\hline Parenchymal hemorrhage & $27[75]$ & 12 [67] & 15 [83] & \\
\hline
\end{tabular}

${ }^{*}$, coronary heart disease, cerebral infarction, pacing surgery. ICH, intracerebral hemorrhage; IQR, interquartile range.

\section{Resumption of anticoagulation}

There were 18 patients who restarted anticoagulation therapy within 3 days after cerebral hemorrhage and 18 patients whose anticoagulant therapy was resumed more than 3 days after hemorrhage. There were no significant differences in gender, age, valvular position, basic disease, type of cerebral hemorrhage, nor anticoagulation intensity before admission between the 2 groups (Table 1). However, patients who underwent surgical intervention resumed anticoagulation therapy earlier than patients who received conservative treatment (Figure 1).

\section{Prognosis}

Table 2 lists the basic characteristics of the patients and the anticoagulant treatment during hospitalization. Patients were followed-up for 12 months or more after discharge. A total of 30 patients survived and 6 died. There was no significant difference in valve replacement site, basic disease, bleeding type, nor interrupted anticoagulation time between patients who survived and those who died (Table 2). The length of hospital stay in the deceased group of patients was significantly longer and that in patients who survived (Figure 2). Of the 6 deceased patients, including 5 males and 1 female, all died due to cerebral parenchymal hemorrhage. Two cases received conservative treatment and 4 cases received surgical treatment. The onset age of deceased patients was higher compared to patients who survived (66 and 59 years, respectively). The interval time between valve replacement and cerebral hemorrhage was shorter $(3 v s$. 6 years) and the anticoagulation intensity before admission was higher (combined anticoagulation was 33\% vs. $7 \%$ ) in deceased patients compared to patients who survived. Of 

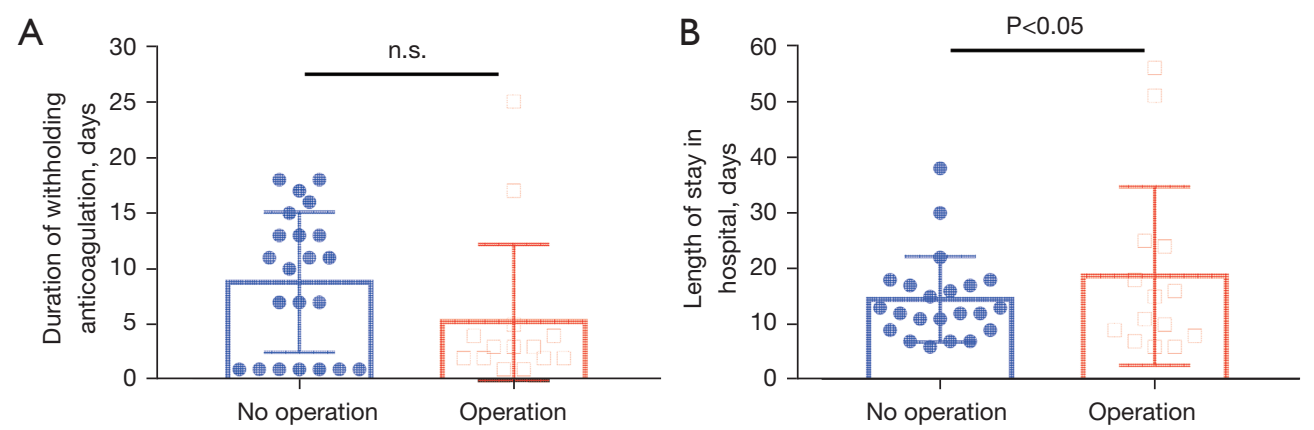

Figure 1 Length of hospital stay and duration of withholding anticoagulation in patients in the operation group and the non-operation group. 'n.s.' means no statistical significance.

Table 2 The basic characteristic of the survival and deceased group of patients after 12 months follow-up

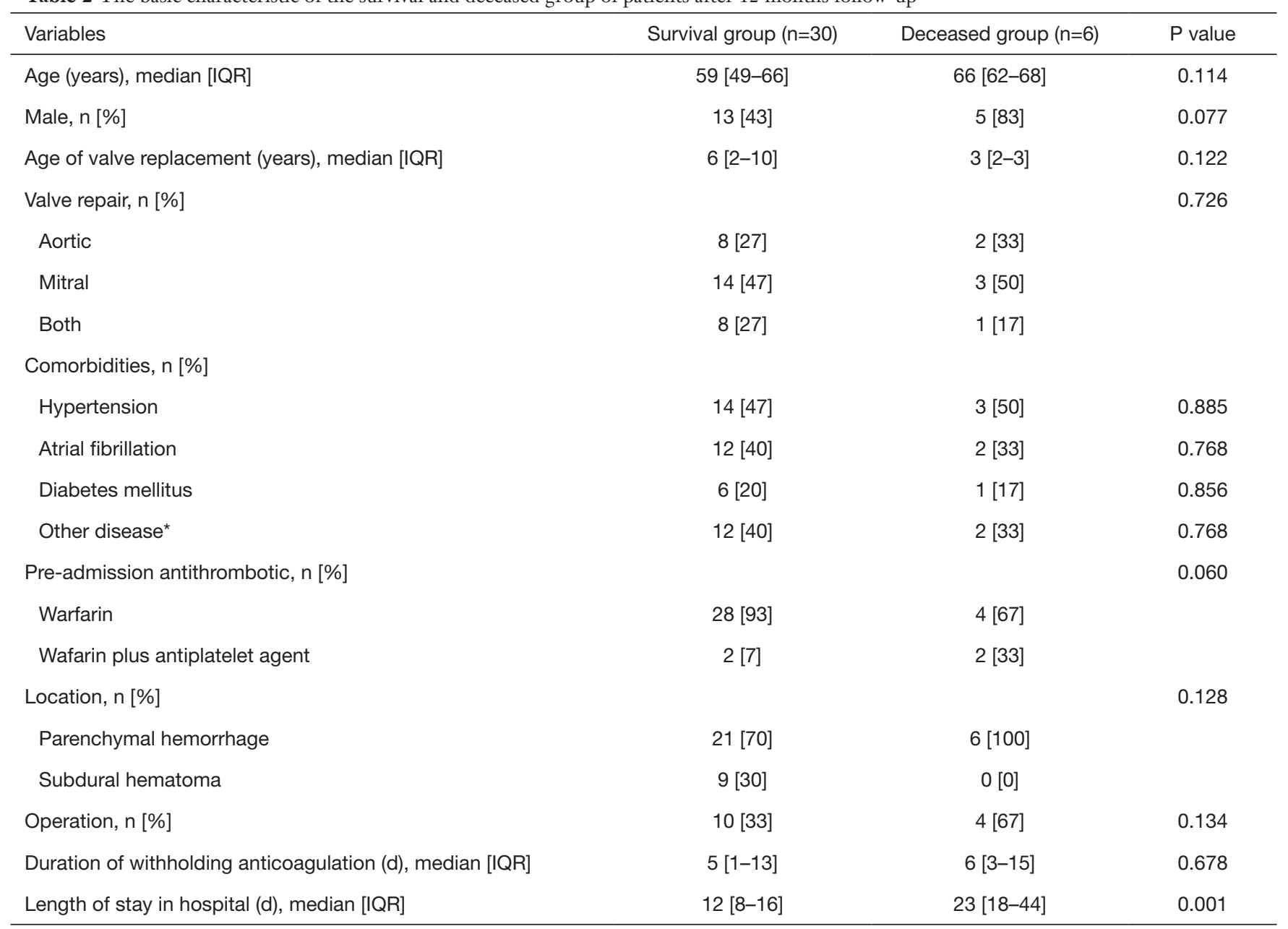

*, coronary heart disease, cerebral infarction, pacing surgery. ICH, intracerebral hemorrhage; IQR, interquartile range.

all the patients followed up, 14 received surgical treatment, $86 \%(12 / 14)$ of whom received surgical intervention within $48 \mathrm{~h}$ after admission. Among the 22 cases that were treated conservatively, the majority $(20 / 22,91 \%)$ presented with cerebral parenchymal hemorrhage. No clinically significant thromboembolism, bleeding recurrence, nor other malignant 
A

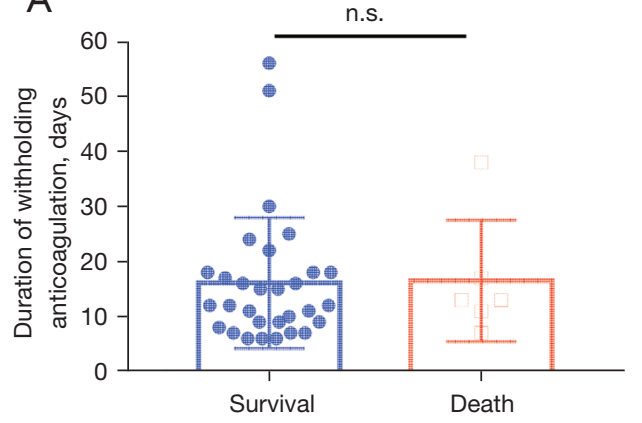

B

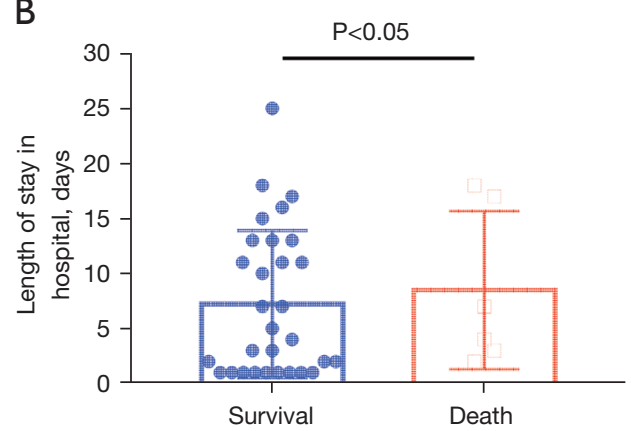

Figure 2 Length of hospital stay and duration of withholding anticoagulation in the survival group and the deceased group. 'n.s.' means no statistical significance.

events were found after the resumption of anticoagulation therapy during hospitalization.

\section{Discussion}

This study retrospectively analyzed the treatment of 40 patients with a history of mechanical heart valve replacement who experienced spontaneous cerebral hemorrhage. Following hemorrhage, the clinical management of such patients must balance the risk of delayed thromboembolism associated with anticoagulation reversal and the risk of hematoma dilation or rebleeding associated with recommencement of anticoagulation. The risk of spontaneous intracranial hemorrhage increases with prolonged oral warfarin treatment time and anticoagulation strength, and indeed, oral warfarin anticoagulation has been associated with a mortality rate of nearly $50 \%$ (3). In this current study, $25 \%(10 / 40)$ of patients with spontaneous cerebral hemorrhage died, $10 \%$ (4/40) died during hospitalization, and $15 \%(6 / 40)$ died from sepsis after discharge. In this study cohort, $78 \%$ (31/40) of intracranial hemorrhage occurred in the intracerebral internal parenchymal, which has been documented to be the most common site (3). The average age of onset was 60 years old, and there was no difference in the incidence of male and female patients. Spontaneous cerebral hemorrhage occurred about 5 years after anticoagulation treatment following valve replacement. The majority of follow-up patients were managed with low levels of warfarin anticoagulation therapy $(1.5-3 \mathrm{mg} /$ day) and the INR was maintained $(1.7-2.2)$. There was no obvious increase in the incidence of bleeding events, nor thromboembolisms, nor any other malignant events.

Wijdicks and colleagues (6) demonstrated that interruption of anticoagulants for 1-2 weeks did not increase the incidence of thromboembolism complications nor recurrence during hospitalization. This concurs with our current data showing that interruption of anticoagulation for an average of 6 days was not associated with increased risk of thromboembolism, nor similar events or complications. Anticoagulant therapy was restarted within 1 week in 22 patients $(61 \%)$, with no bleeding events reported. Other studies have also suggested that early initiation of anticoagulation was associated with a very low risk of increased bleeding $(7,8)$. For patients with artificial valves, heparin anticoagulation can be resumed within 3 days at the earliest, and can be converted to oral anticoagulation in the form of warfarin within 7 days, without the risk of hematoma expansion due to increased bleeding. The timing of restarting anticoagulation therapy should be evaluated individually based on the specific risk factors associated with the valve and the potential causes of bleeding. The risk factors for systemic bleeds, such as previous extracranial bleeds (for example, in the gastrointestinal tract) and other risk factors, including liver and kidney disease, hypertension, and cancer, should also be considered. In this study cohort, there were 36 patients who restarted anticoagulation after hemorrhage, including 17 patients with mitral valve replacement, 10 patients with aortic valve replacement, and 9 patients with mitral valve combined with aortic valve replacement. Among them, there was no difference in the time without anticoagulation therapy, and the location and number of valve replacement did not affect the clinical decision regarding when to restart anticoagulation therapy. Most patients in the study cohort presented with existing comorbidities including hypertension (47\%), atrial fibrillation (39\%), and diabetes (19\%). Recurrent bleeding that occurs before the recommencement of 
anticoagulation therapy may result from inadequate initial blood coagulation. Re-bleeding of the initial hematoma results in growth of the hematoma. The low-density areas, observed on computed tomography (CT) scans, that surround the cerebral hematoma are rich in thrombin, serum inflammatory proteins, and enzymes, and warfarin may prolong the retention effect, resulting in hematoma expansion. Therefore, the decision to resume antithrombotic therapy for the patients depends on the risk of subsequent thromboembolism and recurrent intracranial hemorrhage, and the patient's overall disease status. To date, only a few retrospective case reports have been documented (9-11) and there is currently no consensus on when to resume oral anticoagulation therapy after an intracerebral hemorrhage.

Chandra et al. (7). suggested that it is safe to stop anticoagulant drugs for 7-14 days after the occurrence of an intracerebral hemorrhage, and then restart oral anticoagulant therapy. In the absence of guidelines and more evidence-based medicine, decisions are often made based on past experience or individual considerations. Based on our experience, early surgical hemostasis, postoperative control of blood pressure, and maintenance low INR (1.7-2.0) might be appropriate.

\section{Conclusions}

Hemostatic measures might be an important factor to think about resumption of anticoagulation. While the patient's individual risk factors should be considered, it appears safe to halt anticoagulation therapy for 3 to 7 days after hemorrhage, with administration of low molecular heparin as a bridge treatment within 3 days and warfarin anticoagulant therapy within 1 week. It is also recommended that multidisciplinary teams (including cardiovascular surgery, hematology, and neurosurgery) be invited to assist in making effective and appropriate management decisions for the relaunch of anticoagulation management in these cases.

\section{Study limitations}

There were some limitations to this study. This was a single-center retrospective study, involving only 40 patients, and the decision for anticoagulation reversal was dependent on the experience of the attending neurosurgical doctor. Further multi-centered studies are warranted to confirm verify these results and to evaluate the risk of bleeding and thrombosis in such patients.

\section{Acknowledgments}

Funding: None.

\section{Footnote}

Reporting Checklist: The authors have completed the STROBE reporting checklist. Available at https://atm. amegroups.com/article/view/10.21037/atm-21-6848/rc

Data Sharing Statement: Available at https://atm.amegroups. com/article/view/10.21037/atm-21-6848/dss

Conflicts of Interest: All authors have completed the ICMJE uniform disclosure form (available at https://atm. amegroups.com/article/view/10.21037/atm-21-6848/coif). The authors have no conflicts of interest to declare.

Ethical Statement: The authors are accountable for all aspects of the work in ensuring that questions related to the accuracy or integrity of any part of the work are appropriately investigated and resolved. All procedures performed in this study involving human participants were in accordance with the Declaration of Helsinki (as revised in 2013). The study was approved by ethics committee of the Second Affiliated Hospital of Zhejiang University School of medicine (No. 2020-318). Individual consent for this retrospective analysis was waived.

Open Access Statement: This is an Open Access article distributed in accordance with the Creative Commons Attribution-NonCommercial-NoDerivs 4.0 International License (CC BY-NC-ND 4.0), which permits the noncommercial replication and distribution of the article with the strict proviso that no changes or edits are made and the original work is properly cited (including links to both the formal publication through the relevant DOI and the license). See: https://creativecommons.org/licenses/by-nc-nd/4.0/.

\section{References}

1. Salem DN, O'Gara PT, Madias C, et al. Valvular and structural heart disease: American College of Chest Physicians Evidence-Based Clinical Practice Guidelines (8th Edition). Chest 2008;133:593S-629S.

2. Bonow RO, Carabello BA, Chatterjee K, et al. 2008 Focused update incorporated into the ACC/AHA 2006 guidelines for the management of patients with valvular 
heart disease: a report of the American College of Cardiology/American Heart Association Task Force on Practice Guidelines (Writing Committee to Revise the 1998 Guidelines for the Management of Patients With Valvular Heart Disease): endorsed by the Society of Cardiovascular Anesthesiologists, Society for Cardiovascular Angiography and Interventions, and Society of Thoracic Surgeons. Circulation 2008;118:e523-661.

3. Freeman WD, Aguilar MI. Management of warfarinrelated intracerebral hemorrhage. Expert Rev Neurother 2008;8:271-90.

4. Claassen DO, Kazemi N, Zubkov AY, et al. Restarting anticoagulation therapy after warfarin-associated intracerebral hemorrhage. Arch Neurol 2008;65:1313-8.

5. AlKherayf F, Xu Y, Westwick H, et al. Timing of anticoagulant re-initiation following intracerebral hemorrhage in mechanical heart valves: Survey of neurosurgeons and thrombosis experts. Clin Neurol Neurosurg 2017;154:23-7.

6. Wijdicks EF, Schievink WI, Brown RD, et al. The dilemma of discontinuation of anticoagulation therapy for patients with intracranial hemorrhage and mechanical heart valves. Neurosurgery 1998;42:769-73.

7. Chandra D, Gupta A, Grover V, et al. When should you restart anticoagulation in patients who suffer an intracranial bleed who also have a prosthetic valve? Interact Cardiovasc Thorac Surg 2013;16:520-3.

8. AlKherayf F, Xu Y, Gandara E, et al. Timing of vitamin K antagonist re-initiation following intracranial hemorrhage in mechanical heart valves: Systematic review and metaanalysis. Thromb Res 2016;144:152-7.

9. Amin AG, Ng J, Hsu W, et al. Postoperative anticoagulation in patients with mechanical heart valves following surgical treatment of subdural hematomas. Neurocrit Care 2013;19:90-4.

10. Nishimura K, Koga M, Minematsu K, et al. Intracerebral hemorrhage in patients after heart valve replacement. J Neurol Sci 2016;363:195-9.

11. Kuramatsu JB, Sembill JA, Gerner ST, et al. Management of therapeutic anticoagulation in patients with intracerebral haemorrhage and mechanical heart valves. Eur Heart J 2018;39:1709-23.

(English Language Editor: J. Teoh)
Cite this article as: Luo R, Zhai Z, Wu Q, Chen K, Yi H. Resumption of anticoagulation therapy after spontaneous intracerebral hemorrhage with patients mechanical heart valves. Ann Transl Med 2022;10(2):44. doi: 10.21037/atm-21-6848 